\title{
MENSURAÇÃO DE CONSTRUCTOS PSICOEMOCIONAIS E DE AUTOGESTÃO EM SAÚDE EM PACIENTES COM DOENÇAS METABÓLICAS
}

\author{
MEASUREMENT OF PSYCHOEMOCIAL CONSTRUCTS AND HEALTH \\ SELF-MANAGEMENT IN PATIENTS WITH METABOLICS DISEASES
}

\author{
Cristiane Martins Cunha ${ }^{a^{*}}$, Omar Pereira de Almeida-Neto ${ }^{b^{*}}$, Camila Morais de Rodrigues ${ }^{c^{*}}$ \\ crismcunha@ymail.com ${ }^{\mathrm{a}}$, omarpneto@hotmail.com ${ }^{\mathrm{b}}$, camilamrodri@gmail.com ${ }^{\mathrm{c}}$ \\ Faculdade de Medicina da Universidade Federal de Uberlândia - Uberlândia-MG, Brasil ${ }^{*}$
}

Data do recebimento do artigo: 19/12/2017

Data do aceite: $02 / 07 / 2018$

\begin{abstract}
RESUMO
Introdução: $\mathrm{O}$ diabetes mellitus (DM) e a obesidade $(\mathrm{OB})$ são doenças crônicas que repercutem negativamente em diversos aspectos da vida, sendo fundamental que o paciente adote comportamentos saudáveis para autogerir a saúde. Objetivos: Mensurar as variáveis psicoemocionais e de ativação de indivíduos com doenças metabólicas, comparar essas variáveis segundo o tipo de doença e verificar a influência de variáveis sociodemográficas e clínicas na autogestão em saúde. Método: Estudo transversal, de abordagem quantitativa, realizado em um hospital público. Pacientes adultos, com diagnóstico de $\mathrm{DM}$ ou OB, no mínimo 6 meses, responderam os instrumentos avaliativos (constructos psicoemocionais e ativação). Resultados: Participaram do estudo 80 indivíduos, com idade média de 48,39 (DP=14,59) anos e $65 \%$ eram mulheres. Os escores médios foram: 30,81 ( $\mathrm{DP}=5,08)$ para autoestima, 65,05 $(\mathrm{DP}=17,69)$ para estado de saúde, $8,53(\mathrm{DP}=4,75)$ para ansiedade, $6,53(\mathrm{DP}=4,42)$ para depressão e $64,61(15,54)$ para ativação. Indivíduos com DM apresentaram melhor autoestima e ativação, no entanto tiveram mais sintomas de ansiedade que os $\mathrm{OB}(\mathrm{p}<0,05)$. Os indivíduos $\mathrm{OB}$ referiram melhor estado de saúde, apesar de apresentarem índices maiores de sintomas depressivos $(\mathrm{p}<0,05)$. Quanto às correlações, verificou-se que a variável comorbidades correlacionou com todas as medidas $(\mathrm{p}<0,05)$, exceto com a ativação. A variável idade correlacionou apenas com a ansiedade. Conclusões: Nossa amostra apresentou níveis elevados de autoestima, de estado de saúde e de ativação e baixos níveis de ansiedade e depressão. Indivíduos com DM apresentaram melhor autoestima e mais sintomas de ansiedade que os $\mathrm{OB}$; e os $\mathrm{OB}$ apresentaram melhor estado de saúde e mais sintomas depressivos.
\end{abstract}

Palavras-chave: Autocuidado; diabetes mellitus; obesidade; depressão; autoimagem.

\section{ABSTRACT}

Introduction: Diabetes mellitus (DM) and obesity $(\mathrm{OB})$ are chronic diseases that have negative repercussions in several aspects of life, being essential that the patient adopt healthy behaviors to self-manage health. Objectives: To measure the psycho-emotional variables and activation of individuals with metabolic diseases, to compare these variables according to the type of disease and to verify the influence of sociodemographic and clinical variables on health self-management. Method: A cross-sectional, quantitative approach, carried out in a public hospital. Adult patients, with diagnosis of DM or OB, at least 6 months, answered the evaluative instruments (psychoemotional constructs and activation). Results: Participants were 80 individuals, with a mean age of $48.39(\mathrm{SD}=14.59)$ years and $65 \%$ were women. The mean scores were: $30.81(\mathrm{SD}=5.08)$ for self-esteem, $65.05(\mathrm{SD}=17.69)$ for health status, $8.53(\mathrm{SD}=4.75)$ for anxiety, 
6.53 SD = 4.42) for depression and $64.61(15.54)$ for activation. Individuals with DM presented better self-esteem and activation, however they had more anxiety symptoms than $\mathrm{OB}(\mathrm{p}<0.05)$. OB subjects reported better health status, although they presented higher rates of depressive symptoms $(\mathrm{p}<0.05)$. Regarding the correlations, it was verified that the variable comorbidities correlated with all the measures $(\mathrm{p}<0.05)$, except with the activation. The variable age correlated only with anxiety. Conclusions: Our sample had high levels of self-esteem, health status and activation and low levels of anxiety and depression. Individuals with DM had better self-esteem and more anxiety symptoms than OB; and the OB presented better health and more depressive symptoms.

Keywords: Self Care; diabetes mellitus; obesity; depression; self concept. 


\section{Introdução}

As doenças crônicas são multifatoriais e apresentam início gradual. Além de apresentarem longa duração, são suscetíveis a períodos de agudização no decorrer do tempo e, apesar do grande impacto econômico, normalmente não levam à cura definitiva $^{1}$. Essas doenças requerem cuidados e supervisão contínuos, mudanças nos hábitos de vida, pois podem levar a limitações e incapacidades ${ }^{2}$. Dentre as doenças crônicas com maior prevalência no Brasil, destacamos a obesidade e o Diabetes Mellitus (DM), ambos de origem metabólica ${ }^{3}$.

Pesquisas apontam que pacientes com doenças metabólicas, tais como o DM e a obesidade, apresentam transtornos emocionais, sinais de irritabilidade e instabilidade afetiva e sintomas de ansiedade e depressão ${ }^{4}$. Tais situações podem estar relacionadas ao estigma negativo que essas doenças representam e provocam em seus portadores ${ }^{5,6}$.

Mensurar os constructos psicoemocionais (estado de saúde, autoestima, ansiedade e depressão), de pacientes com Diabetes Mellitus e obesidade é de grande valia, pois pode ser um indicador de como esses indivíduos percebem e convivem com os impactos negativos que essas doenças provocam, sobretudo na autogestão em saúde. Com esses dados, é possível realizar um diagnóstico situacional acerca desses constructos subjetivos e traçar estratégias individualizadas que beneficiem os pacientes com Diabetes Mellitus e obesidade.

Os objetivos desse estudo foram mensurar os escores de constructos psicoemocionais (autoestima, de estado de saúde, ansiedade e depressão) e de ativação (autogestão em saúde) em pacientes que apresentam dois tipos de doenças metabólicas, o Diabetes Mellitus e a obesidade. Também propusemos comparar se há diferença estatística desses escores entre os pacientes com Diabetes Mellitus e obesidade e correlacionar as medidas desses constructos com a idade, escolaridade, tempo de diagnóstico, renda e número de comorbidades.

\section{Método}

Uma amostra não probabilística, por conveniência, do tipo consecutiva, foi formada por pacientes em seguimento no ambulatório de endocrinologia de um hospital público de Minas Gerais. Foram convidados a participar aqueles que atendessem os critérios previamente estabelecidos: diagnóstico de doenças metabólicas, há pelo menos 6 meses, idade entre 18 anos a 80 anos, função cognitiva preservada, a qual foi avaliada por um questionário que estima a capacidade discriminatória e de orientação psíquica e mental do indivíduo no tempo e no espaço. Aos pacientes que foram considerados aptos cognitivamente por essa avaliação, eram realizadas as outras etapas desse estudo. Foi adotado como critério de exclusão: indivíduos com história de internação prévia em um período de até um mês.

Após a apresentação dos objetivos do estudo e mediante os devidos esclarecimentos, foi solicitado o consentimento do participante para se iniciar o protocolo metodológico do projeto. Essa pesquisa foi aprovada pelo Comitê de Ética em Pesquisa, conforme parecer consubstanciado final $\mathrm{n}^{\circ}$ 854.042 obtido em 04/11/2014.

Foi aplicada uma sequência de instrumentos avaliativos: caracterização dos dados sócio demográficos e clínicos, as versões validadas para uso no Brasil da "Patient Activation Measure" (PAM13), da Escala Visual Analógica (EVA), do "Hospital Anxiety and Depression Scale" (HADS) e da Escala de Auto-Estima de Rosenberg (EAER).

Realizamos análises descritivas de frequência simples para as variáveis categóricas (sexo, nível de instrução, estado civil, renda mensal etc.), de medidas de tendência central (média) e de variabilidade (desvio padrão) para as variáveis contínuas.

Para a mensuração e análise dos escores dos constructos autoestima, ansiedade e depressão e ativação, utilizamos a estatística descritiva (frequência simples, tendência central e variabilidade). E, para sua interpretação, utilizamos as recomendações citadas pelos autores, em seus estudos de validação para a população brasileira.

Para avaliar a correlação entre as variáveis (idade, escolaridade, tempo de diagnóstico, renda e número de comorbidades) com as medidas de autoestima, ansiedade e depressão e ativação, utilizamos a correlação de Pearson, entre as variáveis de interesse. A magnitude das correlações obtidas adotadas trata-se de valores de correlação abaixo de 0,30 são de pouca aplicabilidade clínica, 
mesmo com significância estatística; valores entre 0,30 e 0,50 indicam moderada correlação e valores maiores que 0,50 , forte correlação clínica.

\section{Resultados}

Dos 80 participantes, 52 (65\%) eram mulheres. A idade média foi de 48,39 anos e eram procedentes do município local. O nível de escolaridade predominante foi o ensino médio, 29 $(36,3 \%)$, e o tempo médio de ensino formal foi de 8,54 anos. Mais da metade dos participantes, $55(68,8 \%)$, referiu-se casado ou viver em união consensual. A maioria dos participantes, $49(61,3 \%)$, referiu ter alguma atividade ocupacional e renda familiar média de 2,99 salários mínimos (Tabela 1).

Tabela 1: Características sociais e demográficas dos pacientes com doenças metabólicas.

\begin{tabular}{|c|c|c|}
\hline Características & n (\%) & Média $(D P)^{\#}\left[\right.$ variação] $^{£}$ \\
\hline \multicolumn{3}{|l|}{ Sexo } \\
\hline Feminino & $52,0(65,0)$ & \\
\hline Masculino & $28,0(35,0)$ & \\
\hline Idade (em anos) & & $48,39(14,59)[18,0-79,0]$ \\
\hline De 18 a 29 & $7,0(8,8)$ & \\
\hline De 30 a 59 & $54,0(67,5)$ & \\
\hline A partir de 60 & $19,0(23,8)$ & \\
\hline \multicolumn{3}{|l|}{ Grau de Escolaridade } \\
\hline Primário & $22,0(27,5)$ & \\
\hline Fundamental & $18,0(22,5)$ & \\
\hline Médio & $29,0(36,3)$ & \\
\hline Superior & $8,0(10,0)$ & \\
\hline Pós-graduação & $3,0(3,8)$ & \\
\hline Tempo estudo formal (anos) & & $8,54(4,01)[1,0-18,0]$ \\
\hline \multicolumn{3}{|l|}{ Estado conjugal } \\
\hline Casado/união consensual & $55,0(68,8)$ & \\
\hline & $25,0(31,2)$ & \\
\hline \multicolumn{3}{|l|}{ Outros* } \\
\hline Renda familiar $(\mathrm{SM}) * *$ & & $2,99(1,79)[1,0-9,7]$ \\
\hline Renda familiar bruta (reais) & & $2170,02(1292,94)[724,00-7000,00]$ \\
\hline Até 1000,00 & $12,0(15,0)$ & \\
\hline 1000,01 a 2000,00 & $33,0(41,3)$ & \\
\hline Acima de 2000,00 & $35,0(43,8)$ & \\
\hline \multicolumn{3}{|l|}{ Ocupação } \\
\hline Inativo & $31,0(38,8)$ & \\
\hline Ativo & $49,0(61,3)$ & \\
\hline
\end{tabular}


Referente às características clínicas dos pacientes avaliados, verificamos que 50 indivíduos foram diagnosticados com Diabetes Mellitus. Os parti- cipantes apresentaram 3,8 comorbidades e uma média do tempo de diagnósticos foi de 10,87 anos (Tabela 2).

Tabela 2: Características clínicas dos 80 pacientes com doenças metabólicas.

\begin{tabular}{lll}
\hline Características & n (\%) & Média (DP) ${ }^{\#}{\text { [variação }]^{£}}$ \\
\hline
\end{tabular}

Tipo de Doença

Diabetes Mellitus

Obesidade

Número de comorbidades

Até 3

Mais de 3

Tempo de diagnóstico (anos)

Até 2

Mais de 2
$50,0(62,5)$

$30,0(37,5)$
$12,0(15,0)$

$68,0(85,0)$

$80,0(100,0)$

$6,0(7,5)$

$74,0(92,5)$
No que diz respeito às pontuações obtidas pelos instrumentos de medida nos pacientes com doenças metabólicas, verificamos que os pacientes apresentaram um escore médio de autoestima de 30,81 . Os escores para mensuração dos sintomas de ansiedade e depressão evidenciaram que esses sintomas apresen- tam-se em níveis normais, equivalentes ao da população geral. Na avaliação do estado de saúde, os participantes apresentaram escores altos, com valores médios de 65,05 pontos. As pontuações de ativação evidenciaram que os pacientes avaliados apresentaram uma pontuação de 64,61 pontos (Tabela 3).

Tabela 3: Escores médios totais e desvios-padrão (DP) das medidas de autoestima, estado de saúde, ansiedade e depressão e ativação de pacientes com doenças metabólicas.

\begin{tabular}{lll}
\hline \multirow{2}{*}{ Instrumento } & \multicolumn{2}{l}{ Doenças Metabólicas } \\
\cline { 2 - 3 } & Variação & Média (DP) \\
\hline EAER & $21,0-40,0$ & $30,81(5,08)$ \\
EVA & $20,0-100,0$ & $65,05(17,69)$ \\
HADS A & $0-19,0$ & $8,53(4,75)$ \\
HADS D & $0-20,0$ & $6,53(4,42)$ \\
PAM13 & $34,7-100,0$ & $64,61(15,54)$ \\
\hline
\end{tabular}

PAM13 - Patient Activation Measure; EAER - Escala de Auto-Estima de Rosenberg; EVA -Escala visual analógica; HADS A - subescala ansiedade; HADS D- subescala depressão.

Ao avaliarmos se havia diferença entre as pontuações médias das medidas autoestima, estado de saúde, ansiedade, depressão e ativação, observamos que o tipo de doença metabólica interfere nas pontuações nos pacientes avaliados, visto que todas as medidas tiveram diferença estatística $(\mathrm{p}<0,001)$ (Tabela 4). 
Tabela 4: Escores médios totais e desvios-padrão (DP) das medidas de autoestima, estado de saúde, ansiedade e depressão e ativação, conforme o tipo de doença metabólica.

\begin{tabular}{lccc}
\hline \multirow{2}{*}{ Instrumento } & \multicolumn{2}{c}{ Diabetes Mellitus } & \multicolumn{1}{c}{ Obesidade } \\
\cline { 3 - 4 } & \multicolumn{1}{c}{ Média (DP) [Variação] } & 0,000 \\
\cline { 3 - 4 } EAER & $31,0(4,95)[21,0-40,0]$ & $30,5(5,37)[21,0-38,0]$ & 0,000 \\
EVA & $64,98(16,70)[30,0-100,0]$ & $65,17(19,54)[20,0-100,0]$ & 0,000 \\
HADS A & $8,54(5,13)[0-19,0]$ & $8,53(4,15)[2,0-19]$ & 0,000 \\
HADS D & $6,20(4,11)[0-16,0]$ & $7,10(4,92)[0-20,0]$ & 0,000 \\
PAM13 & $65,84(16,83)[34,7-100]$ & $62,58(13,15)[40,1-82,8]$ & \\
PAM13 - Patient Activation Measure; EAER - Escala de Auto-Estima de Rosenberg; EVA -Escala visual \\
analógica; HADS A - subescala ansiedade; HADS D- subescala depressão.
\end{tabular}

Além disso, avaliamos a validade de constructo convergente das medidas com o constructo ativação. Verificamos que a ativação apresenta relação teórica significativa, de moderada magnitude, apenas com a medida de autoestima $(\mathrm{p}<0,05)$ (Tabela 5).

Tabela 5: Coeficientes de Correlação de Pearson entre medidas ativação e as medidas de autoestima, estado de saúde, ansiedade e depressão de pacientes com doenças metabólicas.

\begin{tabular}{lcc}
\hline \multirow{2}{*}{ Instrumentos } & \multicolumn{2}{c}{ PAM13 } \\
\cline { 2 - 3 } & $\mathbf{r}$ & $\mathbf{p}$ \\
\hline EAER & 0,344 & 0,002 \\
HADS A & $-0,146$ & 0,195 \\
HADS D & $-0,116$ & 0,306 \\
EVA & 0,160 & 0,157
\end{tabular}

PAM13 - Patient Activation Measure; EAER - Escala de Auto-Estima de Rosenberg; EVA -Escala visual analógica; HADS A - subescala ansiedade; HADS D- subescala depressão.

A tabela 6 apresenta os Coeficientes de Correlação de Pearson entre as medidas avaliadas com alguns grupos conhecidos. Na amostra estudada, a variável comorbidades apresentou correlação, de fraca a moderada magnitude, com as outras medidas $(\mathrm{p}<0,05)$, exceto com a ativação (autogestão da saúde). E a variável idade correlacionou-se de forma fraca e significativa com a ansiedade (Tabela 6).

Tabela 6: Coeficientes de Correlação de Pearson entre as medidas de ativação, autoestima, estado de saúde, ansiedade e depressão em pacientes com doenças metabólicas, segundo grupos conhecidos.

\begin{tabular}{llllll}
\hline Variável & PAM13 $^{1}$ & EAER $^{2}$ & $\mathbf{E V A}^{\mathbf{3}}$ & HADS A $^{4}$ & HADS D $^{\mathbf{5}}$ \\
\hline Idade (em anos) & 0,042 & 0,176 & 0,144 & $-0,232^{*}$ & $-0,109$ \\
Escolaridade (em anos) & $-0,025$ & $-0,161$ & $-0,030$ & 0,079 & 0,002 \\
Comorbidades diagnosticadas & $-0,127$ & $-0,328^{* *}$ & $-0,279^{*}$ & $0,358^{* *}$ & $0,336^{* *}$ \\
Tempo diagnóstico (em anos) & 0,219 & 0,190 & $-0,089$ & $-0,081$ & 0,012 \\
Renda (em reais) & 0,083 & 0,202 & $-0,018$ & $-0,138$ & $-0,113$ \\
\hline
\end{tabular}

${ }^{\star} \mathrm{p}<0,05 ;{ }^{* *} \mathrm{p}<0,001 .{ }^{1}$ Patient Activation Measure; ${ }^{2}$ Escala de Auto-Estima de Rosenberg; ${ }^{3}$ Escala visual analógica; ${ }^{4}$ subescala ansiedade da HADS; ${ }^{5}$ subescala depressão da HADS. 
Avaliamos a confiabilidade dos instrumentos pelo coeficiente alfa de Cronbach. Todos os instrumentos apresentaram-se confiáveis: PAM13 $(\alpha=0,826)$, EAER $(\alpha=0,669)$ e HADS $(\alpha=0,883)$.

\section{Discussão}

Quanto à amostra, verificamos maior incidência em mulheres e adultos, com faixa etária de 30 a 59 anos, semelhante aos resultados de outro estu$\mathrm{do}^{7}$. Dentre os fatores de risco para doenças crônicas não transmissíveis, a idade é hoje classificada como um fator não modificável, visto que o processo de envelhecimento é diretamente ligado ao risco do desenvolvimento dessas enfermidades ${ }^{8}$.

No que se refere ao estado civil, quase $70 \%$ dos entrevistados declararam ser casados ou estarem em uma união consensual, predominância também relatada por outros autores ${ }^{4,7}$. O estado civil está ligado à incidência de doenças crônicas e com o desenvolvimento de comorbidades e complicações, uma vez que indivíduos que têm a presença de um companheiro apresentaram menores índices de depressão e ansiedade, maior autoestima, maturidade psicológica e melhor autogestão de saúde, quando comparados a pessoas solteiras sem parceiros ${ }^{7}$.

O grau de escolaridade mais prevalente entre os indivíduos entrevistados foi o nível médio, em 29 (36,3\%) dos participantes, tal como encontrado por Porcu et $\mathrm{al}^{4}$. Quando baixo, o nível de escolaridade pode interferir na compreensão do indivíduo quanto às informações que lhe são passadas acerca do cuidado à saúde, seja pelo comprometimento da sua escrita, leitura ou do seu entendimento. Pode também agravar o quadro de saúde, caso o sujeito não valorize as ações preventivas e retarde a procura por assistência médica9 .

Neste estudo, foi constatado que os pacientes tinham uma renda familiar mediana, no limiar de três salários mínimos. Consideramos essa informação importante, uma vez que $61,3 \%$ dos entrevistados afirmaram realizar atividades laborais. Doenças metabólicas crônicas, de acordo com a sua gravidade, podem prejudicar, chegando até mesmo incapacitar sua produtividade profissional e o seu respectivo salário ${ }^{5}$. Portanto, em consequência do seu rendimento, esses indivíduos com doença crônica ficam limitados ou impossibilitados de obter melhores condições de tratamentos e acompanhamentos de saúde.

Quarenta e nove pacientes referiram ter alguma atividade ocupacional, corroborando com os dados de outros autores ${ }^{7,10}$. Manter-se ativo pode refletir maior autonomia e bom estado de saúde do indivíduo; entretanto, o quadro clínico de cada paciente e a sua ocupação devem ser cuidadosamente analisados, a fim de detectar possíveis condições nocivas e adversidades laborais, visando evitar agravos no desenvolvimento das doenças crônicas e de comorbidades.

Entre as duas doenças crônicas abordadas neste estudo, a diabetes mellitus e a obesidade, a de maior incidência entre os pacientes entrevistados foi a primeira, diagnosticada em 50 (62,5\%) dos participantes. Entretanto, é necessário nos atentarmos para o número significativo de obesos descrito, uma vez que a obesidade é um fator de risco importante de evolução para a diabetes mellitus, cuja morbimortalidade é potencializada pelo excesso de peso, principalmente quando associada a doenças cardiovasculares ${ }^{11}$.

Quase totalidade dos participantes (92,5\%) tinham diagnóstico da doença metabólica há mais de 2 anos, o que vai ao encontro de outras pesquisas $^{10,12}$. O período de convivência do indivíduo com a morbidade pode afetar diretamente seu quadro de saúde e o tratamento, uma vez que, no decorrer do tempo, ele tende a compreendê-la e a lidar melhor com as suas peculiaridades e intervenções necessárias ${ }^{13}$.

Em relação às pontuações de autoestima obtidas pela EAER, foi verificado um escore médio elevado, que refletiu em elevada autoestima, resultado encontrado também em outra pesquisa ${ }^{10}$. Em pacientes diabéticos, é importante que a autoestima seja sempre estimulada, pois ela representa um aspecto bastante no autogerenciamento de saúde; uma autoestima elevada influencia o indivíduo a adotar melhores práticas de autocuidado, como adesão a alimentação mais saudável e atividades físicas, controle da glicemia, e, assim, ele apresentará resultados satisfatórios, com domínio e estabilização da doença ${ }^{14}$. Em casos de obesidade, a autoestima pode ser um constructo delicado, uma vez que os atuais padrões de beleza são baseados na magreza, fazendo com que a pessoa obesa se 
sinta estressada, abatida, frustrada, inferior e rejeitada socialmente, por não alcançar as expectativas estéticas impostas pela sociedade. Como consequência, o indivíduo não cuida dos elementos que preservam a saúde, como uma alimentação saudável, exercícios físicos e cuidados com o corpo e aparência; além disso, decorrente da discriminação e do preconceito aos quais é exposto, ele acaba por sofrer pela confiança insuficiente e pelo isolamento social. Todos esses fatores contribuem para o declínio da sua qualidade de vida ${ }^{6}$.

Neste estudo, os escores dos sintomas de ansiedade e depressão em pacientes com doenças metabólicas apresentaram-se em níveis normais, equivalentes com os da população sadia, tal como publicado em um estudo ${ }^{7}$. Indivíduos obesos com DM2 apresentam maior risco de desenvolver ansiedade e depressão do que pessoas com peso normal, porém também diabéticos ${ }^{15}$. $\mathrm{O}$ aumento ponderal pode ser considerado tanto como causa quanto consequência da incidência de distúrbios psicoemocionais, dentre eles a depressão ${ }^{16}$. Enquanto causa, a obesidade induz sofrimento psicológico no indivíduo, por conta dos problemas sociais enfrentados por ele referentes a discriminação, preconceito, estigmas e falta de aceitação $0^{6}$, além da diminuição da autoestima e do bem-es$\operatorname{tar}^{5}$. Uma vez associada ao excesso de peso, a depressão também interfere negativamente nas atividades do obeso, tais como no trabalho, no lazer, nas relações interpessoais e afetivas, prejudicando a sua qualidade de vida ${ }^{5}$ e expectativa de vida ${ }^{6}$.

$\mathrm{O}$ alto nível de ativação constatado nos pacientes deste estudo corrobora com as pesquisas de anteriores ${ }^{17,18}$. As elevadas pontuações de ativação demonstraram que esses pacientes apresentam amplo conhecimento sobre sua condição de saúde, a confiança para agir e as habilidades necessárias para realizar comportamentos e mudanças saudáveis no seu estilo de vida. Deste modo, os pacientes com alto nível de ativação conseguem manter a sua saúde e lidar com os problemas de sua saúde, que neste caso específicos, são advindos da doença. $\mathrm{O}$ envolvimento ativo do paciente na gestão da sua própria saúde é crucial para alcançar os resultados almejados, tendo em vista que, desse modo, ele adquirirá mais experiência em seu cuidado; como consequência, seu quadro de saúde terá uma evolução satisfatória, incluindo a redução dos custos médicos per capita ${ }^{19}$.

Quanto ao estado de saúde, os escores mostraram-se altos, assim como relatado em outra pesquisa $^{5}$. A percepção favorável quanto à própria saúde pode ser justificada pelo fato de que a maior parte dos pacientes abordados neste estudo ainda são ativos, têm condições de cuidar de si mesmos, possuem boa autoestima e não apresentam níveis significativos de ansiedade e depressão. Além disso, em muitos casos a evolução das doenças metabólicas é silenciosa, sem sinais e sintomas desagradáveis nos primeiros anos; consequentemente, a princípio, o indivíduo não sente os efeitos negativos que essas doenças provocam.

Houve diferença nos escores de autoestima entre os pacientes de Diabetes Mellitus e obesidade, uma vez que os diabéticos apresentaram maior nível que os obesos; resultado semelhante aos encontrados por outros autores ${ }^{10,6}$. Devido à alteração da imagem corporal que o aumento de peso provoca, pode ocorrer um abalo na autoestima da pessoa ${ }^{5}$; ela se sente menos atrativa e satisfeita, com menor agilidade, não aceita pela a sociedade, o que gera maior insegurança e sentimento de culpa $^{20}$. No caso da diabetes, muitas vezes o indivíduo tem a sua autoestima fortalecida através da mudança do estilo de vida, baseada na crença em suas próprias capacidades e no seu esforço para realizar o tratamento proposto, assim como para fazer suas escolhas conscientemente ${ }^{21}$.

Com relação ao estado de saúde, os indivíduos com obesidade obtiveram maiores escores do que os com diabetes, resultado concordante com estudo prévio ${ }^{22}$. O fato dos pacientes deste estudo apresentarem boas percepções sobre seu estado de saúde pode ser relacionado ao fato de que a maioria dos participantes conviverem com a condição crônica há muitos anos e, ao longo do tempo, foram adquirindo experiências de aceitação e de autocontrole à nova condição fazendo com que seus portadores passassem a perceber a doença e o impacto dela em suas vidas com menos medos, inseguranças e preocupações ${ }^{23}$.

Enquanto os pacientes obesos apresentaram mais sintomas de depressão, os diabéticos tiveram maior nível de ansiedade, tal como relatado por outros autores ${ }^{6}$. A relação entre obesidade e ansiedade e depressão pode ser considerada como 
sendo bidirecional: enquanto o sobrepeso e a obesidade podem desencadear distúrbios emocionais, a exemplo da depressão e da ansiedade, estas podem alterar o comportamento do indivíduo e causar o seu aumento de peso ${ }^{16}$.

Os pacientes diabéticos apresentaram maior nível de ativação do que os obesos, corroborando com os achados de diversos estudos ${ }^{17,18,24}$. Elevados índices de ativação estão associados a maior realização de comportamentos saudáveis e a melhor facilidade na autogestão em saúde de pacientes diabéticos ${ }^{18}$.

Nesse estudo, a ativação apresentou relação moderada com a medida de autoestima, em acordo com dados encontrados por uma pesquisa precedente $^{25}$. A autoestima tem papel importante no autocuidado, uma vez que motiva o indivíduo a promover mudanças de comportamento benéficas à sua saúde ${ }^{26}$. No tratamento do diabetes, a autoestima do paciente possui relação com a alimentação e com a prática de atividades físicas ${ }^{14}$; nos casos de obesidade, a baixa autoeficácia, motivação insuficiente e outros transtornos psicológicos, dificultam a adoção efetiva a mudanças no estilo de vida ${ }^{27}$.

A idade apresentou correlação inversa significativa de magnitude fraca com a medida de ansiedade; resultado obtido, também, por outros pesquisadores $^{12}$. O fato de o indivíduo mostrar-se menos ansioso com o avanço da idade pode ser justificado pela menor carga de responsabilidades que acompanha o processo do envelhecer; uma vez na fase adulta, a pessoa possui preocupações referentes a trabalho, produtividade, renda salarial e encargos familiares e sociais, podendo desenvolver e agravar quadros de ansiedade, especialmente em situações que envolvam morbidades recém-diagnosticadas ${ }^{28}$.

Referente às comorbidades, essas apresentaram correlação inversa de magnitude moderada com a medida de autoestima. Embora ainda não haja trabalhos na literatura que argumentem essa variável até o momento, e com base nos aspectos da autoestima anteriormente discorridos, acreditamos que a influência das comorbidades na autoestima dos pacientes com doenças metabólicas possa vir a ser negativa, uma vez que a presença de uma ou mais doenças acarreta transtornos psicológicos, físicos, sociais e econômicos a quem as possui. Esta mesma justificativa pode ser aplicada quanto a relação significativa que a variável comorbidades também apresentou com as medidas de ansiedade e depressão.

Apesar de este estudo ter atingido os objetivos propostos, reconhecemos a existência de algumas limitações (tamanho da amostra pequeno, ausência de intervenção clínica para mensurar os efeitos das intervenções e a necessidade de avaliação clínica mais rigorosa e objetiva, baseando-se em variáveis clínicas e resultados de exames propedêuticos ou laboratoriais específicos). Por outro lado, essas limitações podem ser superadas com a realização de novos estudos, preferencialmente longitudinais, baseando-se na aplicação de intervenções em saúde específicas para essas condições de saúde. Mesmo assim, constatamos que nossos resultados são bastante importantes, pois agregam novos indícios na literatura, que até então eram desconhecidos pela comunidade científica.

\section{Conclusão}

Neste estudo, os pacientes acometidos por Diabetes Mellitus e/ou obesidade apresentaram escores altos para as medidas de autoestima, estado de saúde e ativação; referente à ansiedade e à depressão, os níveis mostraram-se equivalentes aos da população geral. O tipo de doença metabólica interferiu significativamente nas pontuações das medidas avaliadas, de modo que indivíduos diabéticos apresentaram melhor autoestima e mais sintomas de ansiedade do que em pacientes obesos, enquanto indivíduos obesos apresentaram melhor percepção do estado de saúde e mais sintomas depressivos do que os diabéticos. A variável comorbidades apresentou correlação de fraca a moderada magnitude com as medidas de autoestima, ansiedade, depressão e estado de saúde; a variável idade, por sua vez, apresentou correlação de fraca magnitude com os sintomas de ansiedade.

Os resultados encontrados são evidências para que os profissionais de saúde englobem a avaliação desses constructos subjetivos na avaliação clínica dos pacientes com doenças metabólicas, e assim busquem estratégias para detectar e reduzir os sintomas depressivos e melhorar a autoestima desses pacientes. Todas essas estratégias visam 
ganhos na ativação do indivíduo com diabetes mellitus e obesidade, baseados no seu autocuidado, na maior disposição e na aptidão do indivíduo na autogestão de sua condição crônica, a fim de obterem melhores resultados em saúde.

\section{Agradecimentos}

Agradecimento à Profa. Dra. Cristiane Martins Cunha, por gentilmente disponibilizar parte dos dados remanescentes de sua pesquisa de doutorado (a qual foi intitulada de "Patient Activation Measure (PAM): Adaptação e validação das versões de 22 e de 13 itens em uma amostra de brasileiros com doenças crônicas") para a elaboração deste trabalho. E também para a Coordenação de Aperfeiçoamento de Pessoal de nível Superior (CAPES) pelo apoio financeiro.

\section{Referências}

1. Brasil. Ministério da Saúde. Secretaria de Atenção à Saúde. Departamento de Atenção Básica. Estratégias para o cuidado da pessoa com doença crônica: obesidade. Brasília; 2014. 212 p.

2. Malta DC, Silva Junior JB. O Plano de Ações Estratégicas para o Enfrentamento das Doenças Crônicas Não Transmissíveis no Brasil e a definição das metas globais para o enfrentamento dessas doenças até 2025: uma revisão. Epidemiol. Serv. Saúde 2013; 22(1): 151-164.

3. Brasil. Ministério da Saúde. Secretaria de Atenção à Saúde. Documento de diretrizes para o cuidado das pessoas com doenças crônicas nas redes de atenção à saúde e nas linhas de cuidado prioritárias. Brasília; 2013.

4. Porcu M, Franzin R, Abreu PB, Previdelli ITS, Astolfi, M. Prevalência de transtornos depressivos e de ansiedade em pacientes obesos submetidos à cirurgia bariátrica. Acta sci., Health sci 2011; 33 (2): 165-171, 2011.

5. Silva SMB, Sintomas de ansiedade, depressão, compulsão alimentar e qualidade de vida em mulheres com obesidade, [Dissertação de Mestrado]. Salvador: Escola Bahiana de Medicina e Saúde Pública; 2015. 59 p.

6. Lima ACR, Oliveira, A. B. Fatores psicológicos da obesidade e alguns apontamentos sobre a terapia cognitivo-comportamental. Mudanças 2016; 24(1): 16 p.

7. Rocha C, Costa E. Aspectos psicológicos na obesidade mórbida: Avaliação dos níveis de ansiedade, depressão e do auto-conceito em obesos que vão ser submetidos à cirurgia bariátrica. Anál. psicol 2012; 4: 451-466.
8. Botrel TA, Costa RD, Costa MD, Costa AMD. Doenças cardiovasculares: causas e prevenção. Rev. bras. clín 2000: 26(3): 87-90

9. Pace AE, Ochoa-Vigo K, Caliri MHL, Fernandes APM. O conhecimento sobre diabetes mellitus no processo de autocuidado. Rev. Latino-Am. Enfermagem 2006; 14(5): 728-734.

10. Fuscaldi FS, Balsanelli ACS, Grossi SAA. Lócus de controle em saúde e autoestima em portadores de diabetes mellitus tipo 2. Rev. Esc. Enferm. USP 2011; 45(4): 855-861.

11. Gomes MB, Netoll DG, Medonça E, Tambascia MA, Fonseca RM, Réa RR et al. Prevalência de sobrepeso e obesidade em pacientes com diabetes mellitus do tipo 2 no Brasil: estudo multicêntrico nacional. Arq Bras Endocrinol Metab 2006; 50(1): 136-144.

12. Starkstein SE, Davis WA, Dragovic M, Cetrullo V, Davis TME, Bruce DG. Diagnostic Criteria for Depression in Type 2 Diabetes: A Data-Driven Approach. Plos One. 2014; 9.

13. Francioni FF, Compreendendo o processo de viver com diabetes mellitus em uma comunidade de Florianópolis, Santa Catarina: das práticas de autoatenção ao apoio social, [Tese de Doutorado]. Florianópolis: Universidade Federal de Santa Catarina; 2010. 186 p.

14. Johnston-Brooks CH, Lewis MA, Garg S. Self-efficacy impacts self-care and $\mathrm{HbAlc}$ in young adults with Type I diabetes. Psychol Med 2002; 64: 43-51.

15. Svenningsson I, Björkelund C, Marklund B, Gedda B. Anxiety and depression in obese and normal-weight individuals with diabetes type 2: A gender perspective. Scand J Caring Sci 2012;26(2): 349-54.

16. Vasques F, Martins FC, Azevedo AP. Aspectos psiquiátricos do tratamento da obesidade. Rev. psiquiatr. clín 2004; 31( 4 ): 195-198.

17. Mayberry R, Willock RJ, Boone L, Lopez P, Qin H, Nicewander D. A High Level of Patient Activation Is Observed But Unrelated to Glycemic Control Among Adults With Type 2 Diabetes. Diabetes Spectr 2010; 23(3): 171-176.

18. Rask KJ, Ziemer DC, Kohler SA, Hawley JN, Arinde FJ, Barnes CS. Patient Activation Is Associated With Healthy Behaviors and Ease in Managing Diabetes in na Indigent Population. Diabetes Educ 2009; 35(4): 622-30.

19. Robert. A. Bailey RA, Pfeifer M, Shillington AC, Harshaw Q, Funnell MM, VanWingen J et al. Effect of a patient decision aid (PDA) for type 2 diabetes on knowledge, decisional self-efficacy, and decisional conflict. BMC Health Serv Res 2016; 16: 14p.

20. Rojas C, Brante M, Miranda E, Pérez-Luco R. Descripción de manifestaciones ansiosas, depresivas y autoconcepto en pacientes obesos mórbidos, sometidos a cirugía bariátrica. Rev. méd. Chile 2011; 139(5): 571-578.

21. Olson, EA, McAuley, E. Impact of a brief intervention on self-regulation, self-efficacy and physical activity in older adults with type 2 diabetes. J Behav Med 2015;38(6): 886-98. 
22. Brown DW, Balluz LS, Giles WH, Beckles GL, Moriarty DG, Ford ES et al. Diabetes mellitus and health-related quality of life among older adults. Findings from the behavioral risk factor surveillance system (BRFSS). Diabetes Res Clin Pract 2004; 65(2): 105-15.

23. 23 Gask L, Macdonald W, Bower P. What is the relationship between diabetes and depression? A qualitative meta-synthesis of patient experience of co-morbidity. Chronic Illness 2011; 7 (3): 239-252.

24. Gomes-Villas Boas LC, Foss MC, Freitas MCF, Pace AE. Relação entre apoio social, adesão aos tratamentos e controle metabólico de pessoas com diabetes mellitus. Rev. Latino-Am. Enfermage. 2012; 20(1): 52-58.

25. Wichit N, Mnatzaganian G, Courtney M, Schulz P, Johnson M. Randomized controlled trial of a Family-oriented self-management program to improve self-efficacy, glycemic control and quality of life among Thai individuals with Type 2 diabetes. Diabetes Res Clin Pract 2017; 123 : 37-48.

26. Jung MJ, Jeong Y. Motivation and Self-Management Behavior of the Individuals With Chronic Low Back Pain Orthopaedic. Nursing 2016; 35(5): 330-337.

27. Vargas V, Elementos para pensar a obesidade, a depressão e a ansiedade [Trabalho de conclusão de curso - Pós-Graduação Lato Sensu]. Ijuí: Universidade Regional do Noroeste do Estado do Rio Grande do Sul; 2012. 17 p.

28. MANETTI ML, Estudo de aspectos profissionais e psicossociais no trabalho e a depressão em enfermeiros atuantes em ambiente hospitalar [Tese de Doutorado]. Ribeirão Preto: Universidade de São Paulo; 2010.

\section{Como citar este artigo:}

Cunha CM, Almeida Neto OP, Rodrigues CM. Mensuração de constructos psicoemocionais e de autogestão em saúde em pacientes com doenças metabólicas. Rev. Aten. Saúde. 2018;16(57):13-23. 